\title{
DIAGNÓSTICO DEL POTENCIAL DE EROSIÓN HIDRÍCA MEDIANTE TÉCNICAS DE GEOPROCESAMIENTO EN LA SUB-CUENCA DEL RÍO ANGASMARCA,LA LIBERTAD, PERU
}

\author{
DIAGNOSIS OF HYDRIC EROSION POTENTIAL USING GEOPROCESSING \\ TECHNIQUES IN SUB-BASIN ANGASMARCA LA LIBERTAD, PERU
}

\author{
${ }^{1}$ Jorge Díaz R
}

\begin{abstract}
Resumen
Las actividades agropecuarias, sin considerar las potencialidades y limitaciones en el uso de la tierra, son una fuente potencial de degradación del medio ambiente, la erosión hídrica es uno de los principales procesos de degradación ambiental. Se realizó un diagnóstico del potencial de erosión hídrica en la sub-cuenca del río Angasmarca, mediante aplicación de la Ecuación Universal de Pérdida de Suelos (USLE) y las técnicas de Geoprocesamiento, que en conjunto se constituyen en una herramienta eficaz para los estudios de este fenómeno. Los factoresde erosiónde USLE objeto de evaluación, la erosividad de las lluvias (R), la erodibilidad del suelo (K), el factor topográfico (LS) y el factor antrópico (CP). La Base de datos fue elaborada con datos de precipitaciones, datos de suelos, datos de topografía, cobertura y uso del suelo a partir de imágenes de satélite, ASTER G-DEM 2, CBERS y la cartografía nacional.

La teledetección y la interface SIG (Sistemas de Información Geográfica), hicieron posible la evaluación de los factores de erosión mencionados y del comportamiento espacial del riesgo potencial de erosión, así como dela elaboración de los mapas respectivos. Del análisis de resultados se encontró que el valor medio anual de la erosividad de las lluvias (EI30), es de 4,76 y la erodibilidad de 0.03412 . Por lo tanto las pérdidas de suelo fueron estimadas por encima de 97.82 en micro cuencas de mayores pendientes y de 77.47 en terrenos con pendientes planas a moderadas. La sub-cuencapresenta en un $47 \%$ de su áreaun elevado riesgo de erosión y en consecuencia a degradación ambiental, vinculado principalmente a alta erosividad de las lluvias y a su relieve muy accidentado, por lo que serecomienda acciones mitigadoras para disminuir los impactos ambientales evitando el uso indiscriminado de estas tierras y el 53\% del área restante deben implementarse labores agrícolas con medidas de conservación de suelos.
\end{abstract}

Palabras-clave: Geoprocesamiento, erosión hídrica, USLE, degradación ambiental.

\begin{abstract}
The Agricultural activities, without considering the potential and limitations on the land use, are a potential source of environmental degradation; water erosion is a major environmental degradation. A diagnosis of potential water erosion in the river sub-basin Angasmarca, by applying Equation Universal Soil Loss (USLE) and geoprocessing techniques, which together constitute an effective tool for studies of this phenomenon. The USLE erosion factors evaluated the rainfall erosivity $(\mathrm{R})$, soil erodibility $(\mathrm{K})$, the topographic factor (LS) and the anthropic factor (CP). The database was developed with rainfall data, soil data, survey data, land cover and land use from satellite imagery, ASTER G-DEM 2, CBERS and national cartography. Remote sensing and GIS interface (Geographic Information Systems), made possible the assessment of erosion factors mentioned and spatial behavior of the potential risk of erosion and the development of the respective maps. The analysis of results was found that the mean annual rainfall erosivity (EI30) is 4,762and erodibility 0.03412 . Therefore soil losses were estimated over 97.82 in micro basins steeper slopes and 77.47 on land with flat to moderate slopes. The sub-basin is about $47 \%$ of the area at high risk of erosion and consequently environmental degradation, mainly linked to high rainfall erosivity and its very rugged relief, so it is recommended mitigating actions to reduce environmental impacts avoiding the indiscriminate use of these lands and the remaining $53 \%$ of the agricultural work should be implemented soil conservation measures.
\end{abstract}

Key words: Geoprocessing, water erosion, USLE, environmental degradation.

\section{Introduccion}

Entre los principales factores relacionados con el aumento de la degradación ambiental están los cambios frecuentesno planificados en el uso dela tierra, por encima de la capacidad de carga del suelo. El manejo inadecuado del suelo, sin límites y riesgos de la degradación ambiental, ha provocado el desarrollo de procesos erosivos acelerados (GUERRA \&CUNHA, 1996). 
La erosión es una de las principales razones por la que los suelos agrícolas pierden su capacidad productiva. La eliminación de la vegetación nativa en una zona determinada provoca el rompimiento, efímero o permanente del equilibrio natural entre el suelo y el medio ambiente. Por regla general, el uso de la tierra para fines agrícolas se inicia con la eliminación de la vegetación nativa, dejando el suelo sin cobertura, por lo que es susceptible a erosión por el agua de lluvia, denominada erosión hídrica (CABRAL et al. 2005).

La Ecuación Universal de Pérdida de Suelos (USLE de la sigla en inglés de Equation Universal Soil Loss), es un modelo empírico válido para estimar y predecir la pérdida de suelos en cuencas hidrográficas (PRUSKI et al., 2006). Siendo las técnicas de Geoprocesamiento herramientas eficaces para los estudios de este fenómeno. Los Sistemas de Información Geográfica (SIG), es la interface que permite determinar el comportamiento espacial del riesgo potencial de áreas con mayor y menor susceptibilidad a erosión, favoreciendo la zonificación ambiental y por consiguiente, la planificación ambiental (FARINASSO, 2005).

La aplicación de modelos matemáticos y de Geoprocesamiento en el análisis de sistemas ambientales son recursos desarrollados que vienen encuadrándose como procedimientos teóricos en el uso de un enfoque holístico a través de evaluaciones cuantitativas y cualitativas. La comprensión de la interacción entre el modelamiento matemático y el uso de los Sistemas de Información Geográfica (SIG), parte de la premisa de que los estudios de series temporales y de análisis espaciales deben ocurrir conjuntamente (CHRISTOFOLETTI, 1999).

El control de la erosión deberá efectuarse a través del uso de acciones preventivas y después de su ocurrencia a través de acciones correctivas y la información sobre el potencial de la tierra para el uso y la ocupación, se debe obtener a través del mapa de riesgos de erosión (RIBEIRO, 2002).

El objetivo general del estudio fue realizar el diagnóstico de degradación ambiental mediante la evaluación del potencial de erosión hídrica enlasub-cuenca del río Angasmarca en la Región La Libertad, siendo objetivos específicos:

1. La estimación de la pérdida de suelos por la erosión hídrica mediante la aplicación de la Ecuación Universal de Pérdida de Suelos (USLE).

2. Determinar del comportamiento espacial del riesgo potencial de erosión y la degradación ambientalen la sub-cuenca, mediante la aplicación delos Sistemas de Información Geográfica, SIG.

3. Proponer acciones mitigadoras para áreas con riesgo de erosión, a fin de que los impactos ambientales de utilización indiscriminada de estas tierras tengan su magnitud reducida.

\section{Materiales y método}

\section{Materiales}

Cartografía base y temáticadel área de estudio. Las cartas topográficas editadas por el Instituto Geográfico Nacional (IGN), escala 1:100.000, con curvas de nivel cada 50 m, las hojas: 16-g, 16-h, 17-g y 17-h, utilizadas en el estudio de redes de drenaje, Modelo Digital del Terreno (MDT), pendientes, ecología, geología y suelos.

Imágenes de sensores remotos.Uso de un mosaico de cuatro imágenes de satélite G-DEM2 del sensor ASTER ASTGTM2: S08W078, S08W079, S09W078 y S09W079, datum WGS84, geoide EGM96, en formato GeoTIFF, resolución espacial de $30 \mathrm{~m}$, georeferenciadas en coordenadas geográficas. Utilizadas en MDT para delimitación de micro cuencas, mapas de pendientes y redes de drenaje. La imagen orbital CBERS2 CCD1XS 20060721_190_109_L2, del 21 de julio de 2006, del sensor CCD a bordo del satélite CBERS-2, del Instituto Nacional de Pesquisas Espaciales (INPE), del Brasil, para obtención del mapa de uso y ocupación del suelo.

Datos de análisis físico-químico de suelos. Obtenidos del estudio: "Inventario, Evaluación y Uso Racional de los Recursos Naturales de las Cuencas Hidrográficas de los Ríos Santa, Lacramarca y Nepeña» ONERN, 1972.

\section{Equipamiento}

Los equipos utilizados en el desarrollo de este trabajo fueron: a) PC PENTIUM 5 con 4 GB de RAM, 500 GB de disco duro SVGA monitor de 19", teclado, impresora de CD/DVD.b) Software: ArcGIS Desktop 9.2 (ESRI), SPRING 5.1.6 (INPE) y Microsoft Office (Excel 2007, Word 2007, etc.).

\section{Método}

El estudio "diagnóstico de degradación ambiental mediante la evaluación deriesgo potencial de erosión hídrica en la sub-cuenca del rio Angasmarca", fue desarrollado mediante aplicación del modelo matemático de la Ecuación Universal de Pérdida de Suelos (USLE). Esta ecuación estimadora de pérdida de suelos es la más utilizada y la más aceptada, elaborada con una metodología que correlaciona la predicción media anual de pérdida de suelos (, causada por la erosión hídrica laminar Se expresa matemáticamente mediante la siguiente ecuación:

$$
A=R \cdot K \cdot L . S . C . P \rightarrow(2.1)
$$

donde: $\mathrm{A}=$ perdida media anual de suelos, ; $\mathrm{R}=$ factor erosividad de la lluvia, en ; $\mathrm{K}=$ factor probabilidad del suelo, en; $\mathrm{L}=$ factor longitud de pendiente o rampa en $\mathrm{m} ; \mathrm{S}$ $=$ factor pendiente o declive y los factores adimensionales $\mathrm{C}=$ factor de uso y manejo del suelo y $\mathrm{P}=$ factor práctica de conservación de suelos. El modelo matemático de 
USLE fue desarrollado mediante aplicación de las técnicas de geoprocesamiento de imágenes orbitales y el uso de Sistemas de Información Geográfica (SIG). El procedimiento se indica en el diagrama de flujo en la Fig.1.

Elaboración del modelo digital del terreno (MDT). Fue elaborado a partir de un mosaico de las imágenes ASTER G-DEM2, mediante las herramientas 3D Analist de ArcGIS, se generó un TIN de contornos. Las fajas altimétricas de TIN se clasificaron en 11 clases con 250 $\mathrm{m}$ de intervalo.
Delimitación de las micro cuencas hidrográficas. La delimitación de la sub-cuenca Angasmarca en micro cuencas fue a través de las herramientas "Watershed Delineation Tools" del programa FWD. A partir del MDT (dato entrada), el programa crea una red de flujo basada en un umbral proporcionado por el usuario y delimita las micro cuencas para cada enlace de la red de flujo. El resultado fue la salida automática de los raster: micro cuencas delimitadas (watershed - delimitation), redes de drenaje (stream), dirección del flujo (flow-direction), flujo de acumulación (flow-Acumulation).

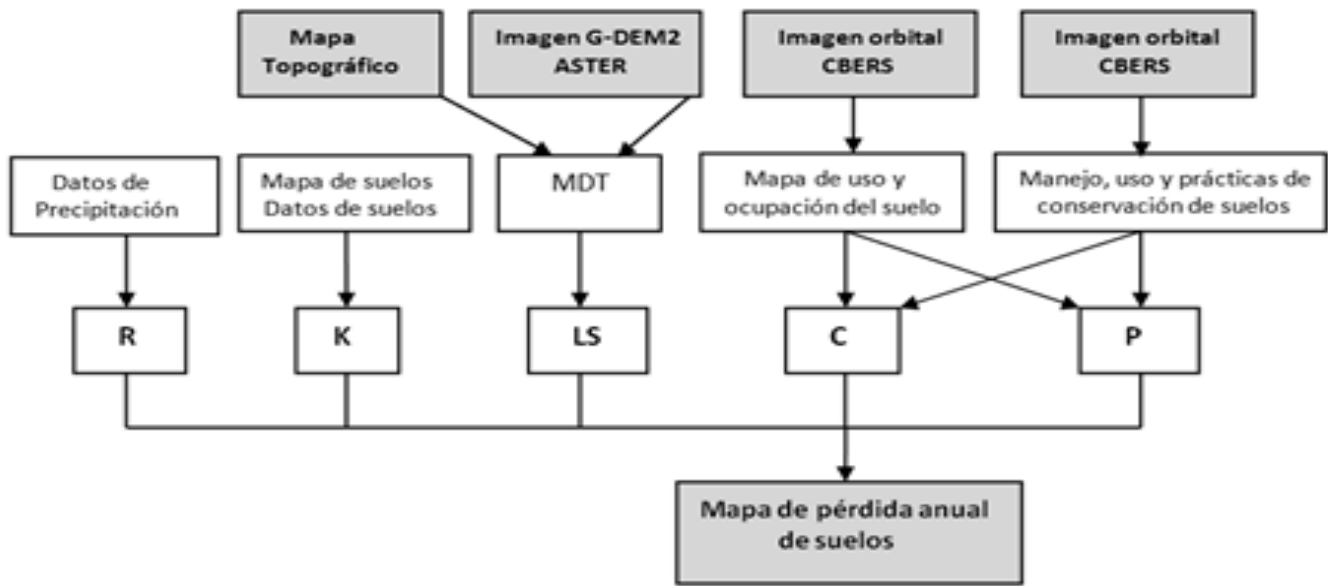

Figura 1. Flujograma simplificado para el cálculo de pérdida de suelos, mediante la Ecuación Universal de Pérdida de Suelos, USLE.

Elaboración de mapas de la sub-cuenca. Mediante el uso de las herramientas deArcGIS, a partir de las imágenes ASTER DEM y las cartas topográficas del IGN, se elaboraron los mapas (layers) en formato shapefile: MDT, pendientes (S), redes de drenaje (RD) y curvas de nivel $(\mathrm{CN})$, así como la confección de tablas de atributos de los layers respectivos.

Clasificación del uso y ocupación del suelo. El mapa de uso y ocupación del suelo (MUO), fue elaborado por clasificación supervisada de imágenes CBERS-2, de composición en color (RGB) 3.4.2 (tres bandas), mediante uso el software SPRING 5.1.6). Las clases de uso se muestran en la Tabla1.

Tabla 1. Clasificación de uso y ocupación del suelo.

\begin{tabular}{cl}
\hline $\mathrm{N}^{0}$ orden & Clase de uso - ocupación \\
\hline 1 & Forestal \\
2 & Pastizal abierto \\
3 & Pastizal cerrado \\
4 & Agricultura de secano \\
5 & Agricultura de irrigación \\
6 & Suelo expuesto \\
7 & agua \\
\hline
\end{tabular}

Estimación de la erosividad de las lluvias (R). El índice de erosividad ( $\mathrm{R}=\mathrm{EI} 30)$, fue obtenido según con criterios propuestos por Bertoni y Lombardi Neto (1993), mediante la aplicación de la ecuación 2.2

$$
\mathrm{El}_{30}=67.355\left(\frac{\mathrm{r}^{2}}{\mathrm{p}}\right)^{0.85}
$$

siendo (r) la precipitación media mensual en mm (P), la precipitación media anual en mm y EI30 es el valor medio mensual del índice de erosión en $\mathrm{MJ} \cdot \mathrm{ha}^{-1} \cdot \mathrm{mm} \cdot \mathrm{h}^{-1}$. mes. Los cálculos de $\mathrm{P}$ se obtuvieron utilizando registros de precipitación mensual de las 3 estaciones con la ayuda del programa MS Excel.

Estimación indirecta de erodibilidad de los suelos (K). La erodibilidad del suelo fue obtenida mediante la aplicación de las ecuaciones de 2.3 y 2.4 elaboradas por Wischmeier y Smith (1978), a partir de los datos de las propiedades físico- mecánicas de suelos de la sub-cuenca y los resultados fueron comprobados con la lectura gráfica del nomograma también de Wischmeier y Smith (1978). Según Bertoni, Lombardi (2010).

$$
K=\left[\frac{2.1 \times 10^{-4}(12-O M) M^{2.24}+3.25(s-2)+2.5(p-3)}{100}\right] 0.1318
$$


donde: $\mathrm{OM}=$ contenido de materia orgánica, dag $\mathrm{kg}^{-1}, \mathrm{M}$ = parámetro que representa la textura del suelo, $\mathrm{s}=$ clase de estructura del suelo, sin dimensiones y $\mathrm{p}=$ perfil de permeabilidad, sin dimensiones. El valor de M se calcula por la ecuación:

$$
M=(\% \text { limo }+\% \text { arenafina })(100-\% \text { arcilla })
$$

Longitud de rampas ( $\mathbf{L})$. La longitud media de rampas (longitud media de pendientes), de las micro cuencas se obtuvo mediante el método del rectángulo equivalente modificado de Vilela \& Mattos (1975), a partir del mapa hidrográfico y aplicación de la ecuación 2.5.

$$
L=A /(4 l)
$$

siendo, $\mathrm{L}=$ longitud de la rampa $(\mathrm{m}), \mathrm{A}=$ área de la cuenca $\left(\mathrm{m}^{2}\right)$; sumatoria de la longitud de todos los cursos de agua de la cuenca $(\mathrm{m})$.

Factor pendiente (S). De acuerdo con la metodología desarrollada por Wischmeier \& Smith (1978), este factor (S), se obtiene mediante la aplicación de la ecuación 2.6. La Tabla 2 muestra la clasificación de las pendientes utilizadas en este estudio.

$$
S=0,00654 s^{2}+0,0456 s+0,065
$$

donde $\mathrm{S}=$ factor de pendiente (adimensional) y $\mathbf{s}=$ pendiente media de la vertiente en porcentaje (\%).

Factor topográfico (factor LS). Factor adimensional obtenido a partir del mapa de pendientes o según la expresión matemática de la ecuación 2.7 .

$$
L S=0,00984 L^{0.63} S^{1.18}
$$

donde: $\mathrm{L}=$ longitud de rampa $(\mathrm{m}), \mathrm{S}=$ pendiente $(\%)$. Esta ecuación supone pendiente esencialmente uniforme, es decir, no tiene en cuenta si es cóncava oconvexa, lo que constituye una limitación del uso de la USLE (PRUSKI, 2009).

Tabla 2. Clasificación de pendientes para el estudio.

\begin{tabular}{cccl}
\hline Clase Símbolo & $\begin{array}{c}\text { Intervalo } \\
(\%)\end{array}$ & Clasificación cualitativa \\
\hline 1 & A & $00-04$ & Plano a ligeramente inclinada \\
2 & B & $04-15$ & $\begin{array}{l}\text { moderada a fuertemente } \\
\text { inclinada }\end{array}$ \\
3 & C & $15-25$ & moderadamente empinada \\
4 & D & $25-50$ & empinado \\
5 & E & $50-75$ & muy empinada \\
6 & F & $75-100$ & $\begin{array}{l}\text { extremadamente muy } \\
\text { empinada }\end{array}$ \\
7 & G & $>100$ & abrupto \\
\hline
\end{tabular}

Fuente: Adaptada del Reglamento de Clasificación de Tierras por Capacidad de Uso Mayor, del Ministério de Agricultura del Perú (D.S. No. 0062-75- AG, 1975).
Potencial natural de erosión (PNE). Calculado a partir de los valores medios de la erosividad de las lluvias (factor R), la erodibilidad del suelo (factor K) y el factor topográfico (LS), mediante la ecuación 2.8 y uso del software de MS Excel.

$$
P N E=\text { R.K.L.S }
$$

Factores uso, manejo y prácticas de conservación de suelos. Los factores C, P y factor CP (factor antrópico), fue obtenido de los valores de uso y ocupación del suelo (MUO), así como de evaluación de prácticas de conservación de suelos sobre la base de los valores propuestos por Righeto (1998), Ruhoff (2004) y Tomazoni et al (2005).

La adopción del valor medio ponderado de CP para agricultura y pastoreo fueron determinada mediante la ecuación 2.9

$$
\mathrm{CP}_{\text {medio }}=\frac{\Sigma(\mathrm{CP} \mathrm{xA})}{\mathrm{AT}}
$$

donde: $\mathrm{CP}=$ valores de las actividades según la Tabla $3, \mathrm{~A}$ = área de ocupación la micro cuenca $(\%), \mathrm{AT}=$ porcentaje total del área de la actividad en micro cuencas.

Tabla 3. Valores de P, C y factor CP para algunos tipos de manejo de suelos adaptados para la Sub-cuenca de Angasmarca.

\begin{tabular}{lccc}
\hline $\begin{array}{c}\text { Clase } \\
\text { Uso de suelo }\end{array}$ & $\begin{array}{c}\text { Valor } \\
\text { de C }\end{array}$ & $\begin{array}{c}\text { Valor } \\
\text { de P }\end{array}$ & $\begin{array}{c}\text { CP } \\
\text { (adim) }\end{array}$ \\
\hline Forestal & 0.012 & 0.1 & 0.0012 \\
Pastizal & $0.01-0.025$ & 0.4 & $0.004-0.0100$ \\
Agricultura de & $0.18-0.290$ & 0.7 & $0.126-0.2030$ \\
secano & & & $0.180-0.3600$ \\
Agricultura & $0.18-0.360$ & 1.0 & 1.0000 \\
irrigada & 1.000 & 1.0 & 1.0000 \\
Suelo expuesto & 1.000 & 1.0 & 0.000 \\
Minería & 0.000 & 0.0 & \\
Agua & \multicolumn{3}{c}{ Fuentes: Righeto (1998), Ruhoff (2004), Tomazoni et al (2005) }
\end{tabular}

Pérdida de suelos por erosión hídrica en la subcuenca. La pérdida de suelos por erosión laminar (A) en (t.ha/año), fue determinada mediante la aplicación de la ecuación 2.1, o como el producto del potencial natural de erosión (PNE) y el factor antrópico (CP), mediante aplicación de la ecuación 2.8.

$$
A=R \cdot K \cdot L S . C P \quad(2.1) \quad \text { ó } A=P N E . C P
$$

Tolerancia límite de la pérdida de suelo. La tolerancia de la pérdida de suelo por la erosión ( $\mathrm{T}$ ) propuesta por Oliveira (2004), correlaciona el efecto de la permeabilidad y el contenido de materia orgánica de las respectivas pérdidas mediante la ecuación 2.10 de la siguiente manera:

$$
T=h r m p 1000^{-1}
$$


donde $\mathrm{T}=$ tolerancia de pérdida de suelos por erosión (t.ha ${ }^{1}$.año $\left.{ }^{-1}\right), h=$ espesor del horizonte $(\mathrm{cm}), r=$ coeficiente que expresa efecto de la relación textural en horizontes Ay Ben ponderación de las pérdidas de suelo $\left(\mathrm{g} \cdot \mathrm{kg}^{-1}\right), \mathrm{m}=$ factor de materia orgánica en capa de 0 a $20 \mathrm{~cm}, \mathrm{p}=$ factor de efecto de permeabilidad de suelo. Los valores de $\mathrm{r}$ son iguales a $1.0,0.9$ y 0.8 para los suelos de contenido de arcilla en el horizonte A, mayor que $40 \%$, entre 40 y $20 \%$ y menor que $20 \%$, respectivamente.

\section{Resultados y discusión}

Factor erosividad (Factor R). Del análisis de datos de precipitaciones según el histograma en la Fig. 2, se encontró que la precipitación media anual es de 755.1 $\mathrm{mm}$, el valor medio mensual más alto en el mes de marzo de $159.1 \mathrm{~mm}$ y el más bajo en el mes de julio de 2.3 $\mathrm{mm}$, la mayor concentración de las lluvias ocurre entre octubre y abril, con un verano lluvioso, donde ocurre el $92.2 \%$ de la precipitación total, mientras que entre mayo a setiembre ocurren $7.8 \%$ de las lluvias.

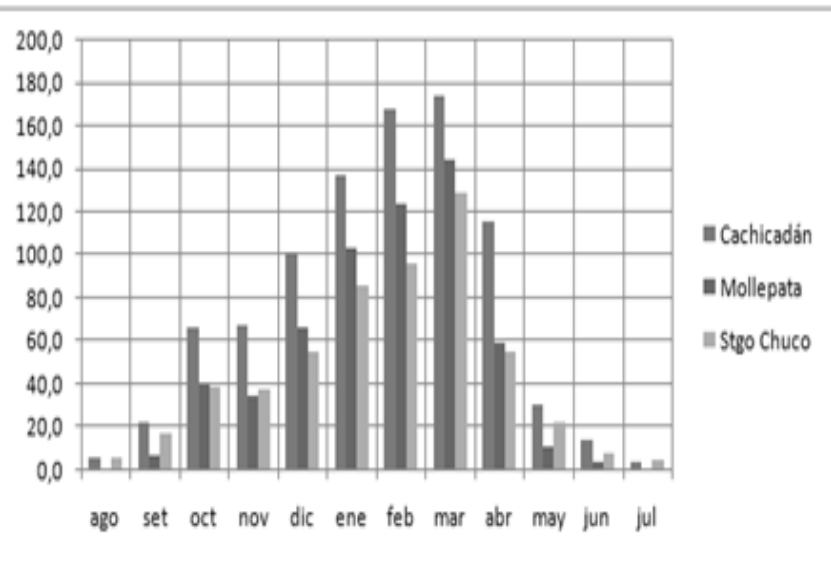

Figura 2. Histograma de la distribución de la precipitación media mensual (mm) de estaciones pluviométricas de la sub-cuenca Angasmarca (1981-2010).

La estación lluviosa coincide con el inicio de la preparación de tierras y la siembra de los cultivos anuales, debiendo tomarse medidas que traten de minimizar las pérdidas de suelo por erosión conadopción de prácticas de conservación. En la Fig.3 se observa que el valor de erosividaden áreas de influencia de las estaciones Mollepata y Cachicadán son de 4,199.2 y 5,324.8MJ.ha${ }^{1} \mathrm{~mm} \cdot \mathrm{h}^{-1}$ respectivamente. Por tanto el factor " $\mathrm{R}$ " medio para USLE fue de 4,762.0 MJ.ha ${ }^{-1} \mathrm{~mm} . \mathrm{h}^{-1}$, donde el 97.8\% de este índice se produjo durante la estación lluviosa y sólo el 2.2\% en la estación seca.

Según la Tabla 4, el período de retorno y la probabilidad de ocurrencia de lluvias para el mayor índice de erosividad anual fue de 31 años y de $3.2 \%$, respectivamente. Por lo tanto, se espera para esta localidad una probabilidad de $3.2 \%$, que el valor del índice de erosividad anual sea igual o superior a 5,324.8 MJ.ha-1 mm.h ${ }^{-1}$, al menos una vez cada 31 años, mientras que en la localidad con tasa más baja de erosividad anual, el valor del período de retorno fue de 7 años, con $14.3 \%$ de probabilidad de ocurrencia de lluvias, se cree, con una probabilidad del 14.3\%, un índice de erosividad anual superior a 3,564.5 MJ.ha ${ }^{1} \mathrm{~mm} \cdot \mathrm{h}^{-1}$ al menos una vez cada 7 años.
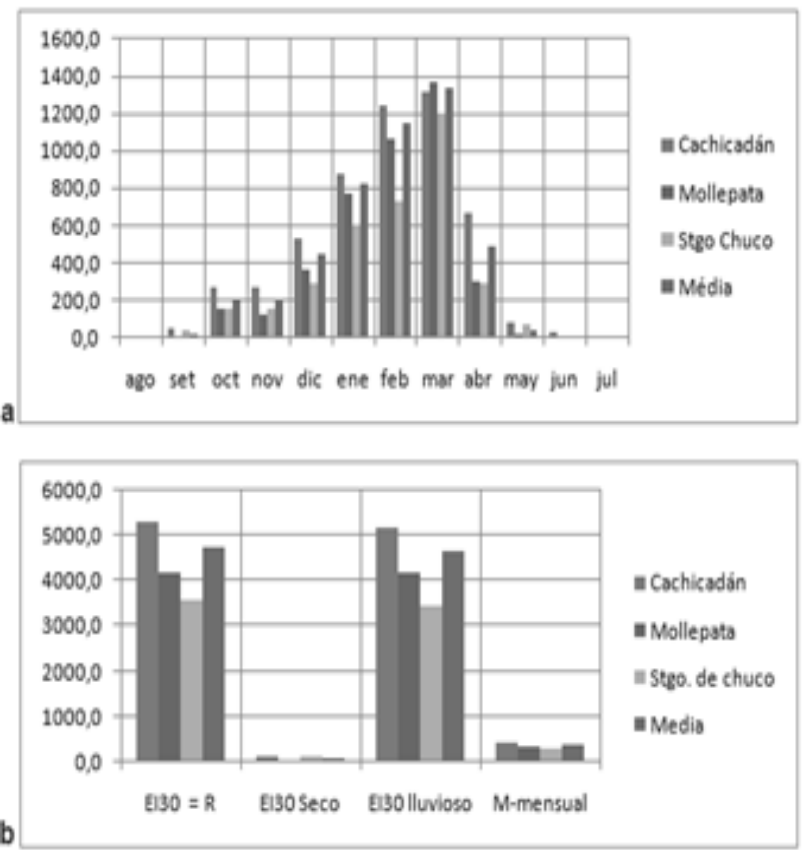

Figura 3. a) Histograma de distribución de la erosividad media mensualde las lluvias, b) valores $\mathrm{EI} 30=\mathrm{R}$, EI30 Seco y EI30 lluvioso de 1981-2010.

Tabla 4. Erosividad media anual y mensual, probabilidad de ocurrencia $(\mathrm{Pr})$ y el período de retorno $(\mathrm{T})$, para el área de estudio en el período 1981-2010.

\begin{tabular}{lccccc}
\hline $\begin{array}{l}\text { Estación } \\
\text { Pluviométrica }\end{array}$ & NO & $\begin{array}{c}\text { MA } \\
(\mathrm{mm})\end{array}$ & $\begin{array}{c}\text { MM } \\
(\mathrm{mm})\end{array}$ & $\mathrm{T}$ & $\mathrm{Pr}$ \\
\hline Cachicadán & 1 & $5,324.8$ & 443.7 & 31.0 & 3.2 \\
Mollepata & 2 & $4,199.2$ & 349.9 & 15.5 & 6.4 \\
$\begin{array}{l}\text { Santiago de } \\
\text { Chuco }\end{array}$ & 3 & $3,564.5$ & 297.0 & 7.0 & 14.3 \\
\hline
\end{tabular}

$\mathrm{NO}=$ número de orden, $\mathrm{MA}=$ media anual, $\mathrm{MM}=$ media mensual, $\mathrm{T}=$ tiempo de retorno, $\operatorname{Pr}=$ probabilidad

Factor erodibilidad del suelo (K). Los valores de erodabilidad $(\mathrm{K})$ determinados, mediante la aplicación de las ecuaciones 2.3 y 2.4 de Wischmeier y Smith (1978), sobre la base de datos del estudio de suelos (Tabla 5), fueron idénticos a los valores obtenidos mediante la lectura gráfica del nomograma de los mismos autores. De los resultados podemos deducir que los suelos con menor erodibilidad fueron los Páramo Andosoles, a diferencia de los Kastanazem que son suelos cálcicos y tienen mayor valor de erodibilidad. 
Modelamiento hidrológico de la sub-cuenca. En la Fig. 4, el modelo digital del terreno MDT, muestralos diferentes niveles altitudinales de la sub-cuenca, varían entre 1,980 a $4,600 \mathrm{msnm}$.
Longitud de rampa (factor $\mathbf{L}$ ). La Tabla 6 muestra los resultados del cálculo de longitud de rampas (L), la longitud media de rampaen la sub-cuenca es de 0,433 $\mathrm{km}$, es posible que exista influencia geológica en estos valores, están en las formaciones Goyllaresquizga (Ki-g) y Chicama (Js-chic), según el mapa geológico (ONERN,1972).

Tabla 5. La erodibilidad de suelos (K), de la sub-cuencaAngasmarca, segúnfórmulas y nomograma de Wischmeier y Smith 1978 (BERTONI \& LOMBARDI NETO, 2010).

\begin{tabular}{|c|c|c|c|c|c|c|c|c|c|c|c|c|}
\hline \multirow{3}{*}{ Símbolo } & \multirow{3}{*}{$\begin{array}{l}\text { Grandes Grupos } \\
\text { deSuelos } \\
\text { (FAO) }\end{array}$} & \multirow{3}{*}{$\begin{array}{l}\text { Hori- } \\
\text { zonte }\end{array}$} & \multicolumn{4}{|c|}{ ANALISE MECANICO } & \multicolumn{5}{|c|}{ PARAMETROS DEL FATOR “K” } & \multirow{3}{*}{ Factor k } \\
\hline & & & $\begin{array}{c}\text { Profun- } \\
\text { didad }\end{array}$ & $\begin{array}{l}\text { Arena } \\
\text { Gruesa }\end{array}$ & $\begin{array}{l}\text { Arena } \\
\text { Fina }\end{array}$ & Limo & & illa OM & $\mathrm{M}$ & $\begin{array}{c}\text { Estruc } \\
\text { tura }\end{array}$ & $\begin{array}{l}\text { Permea- } \\
\text { bilidad }\end{array}$ & \\
\hline & & & $(\mathrm{cm})$ & $(\%)$ & $(\%)$ & $(\%)$ & $(\%)$ & $(\%)$ & Adim. & $\mathrm{s}$ & $\mathrm{p}$ & \\
\hline \multirow[t]{2}{*}{ PeLae-c } & Páramo Andosol & A & $0-30$ & 31 & 21 & 34 & 14.0 & 7.93 & 4.730 & 1 & 2 & 0.00984 \\
\hline & & (B) & $30-50$ & 41 & 27 & 24 & 8.0 & 0.83 & 4.692 & 1 & 2 & 0.03980 \\
\hline \multirow[t]{2}{*}{$\mathrm{Lad}-\mathrm{c}$} & Kastanazem lúvico & A1 & $0-30$ & 30 & 20 & 32 & 18.0 & 3.58 & 4.264 & 2 & 3 & 0.03202 \\
\hline & & B2 & $30-70$ & 24 & 16 & 32 & 28.0 & 0.12 & 3.456 & 3 & 4 & 0.04314 \\
\hline \multirow[t]{3}{*}{ Lad - c } & Kastanazencálcico & A & $0-30$ & 31 & 21 & 28 & 20.0 & 1.52 & 3.920 & 2 & 3 & 0.03621 \\
\hline & & B2 & $30-70$ & 32 & 22 & 24 & 22.0 & 0.89 & 3.588 & 3 & 2 & 0.03569 \\
\hline & & $\mathrm{C}$ & $70-160$ & 32 & 22 & 30 & 16.0 & 0.89 & 4.368 & 4 & 2 & 0.04870 \\
\hline \multirow[t]{3}{*}{ Ld X-c } & Xerosol lúvico & A & $0-15$ & 22 & 14 & 42 & 22.0 & 4 & 4.368 & 2 & 4 & 0.03457 \\
\hline & & B & $15-65$ & 24 & 16 & 32 & 28.0 & 0.89 & 3.240 & 3 & 4 & 0.03847 \\
\hline & & B3 & $65+110$ & 27 & 15 & 30 & 28.0 & 0.89 & 3.240 & 2 & 4 & 0.03419 \\
\hline
\end{tabular}

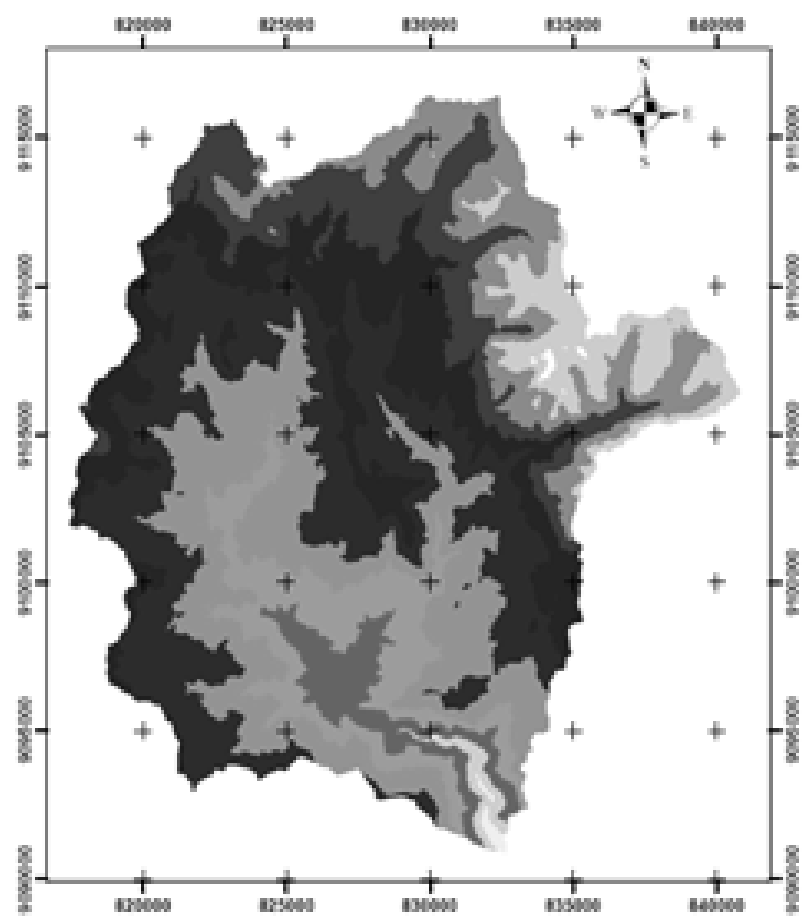

\section{Modelo Digital del Terreno (GDEM 2 -ASTER)}

\section{Sub-cuenca Angasmarca} La Libertad, Peru

Departamemo de Recursos Hidricos FU. UNALM UTM Zens 17 Ser Enals Numeriea tabs be Enesils orifles:

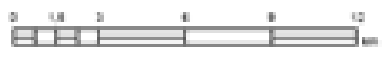

\section{Leyenda: Altitudes (m)}

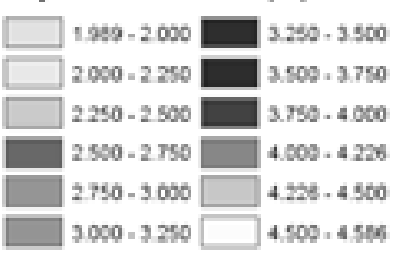

Figura 4. Altimetría - Modelo Digital del Terreno. SIG-ArcGIS 9.2. 
Pendiente o declividad media (Factor S). De acuerdo a los resultados en la Tabla 6 , se observa que los valores más altos de pendientes (por encima del $50 \%$ ), se producen en micro cuencas predominantemente montañosas, en $73.44 \mathrm{~km}^{2}$ (20.80\%), consideradas como pendientes muy pronunciadas. Las otras micro cuencas consideradas empinadas $(25-50 \%$ de pendiente), en $279.64 \mathrm{~km}^{2}$ (79.20\% de la sub-cuenca).

Factor topográfico (Factor LS). Según la Tabla 6 el $80.08 \%$ el factor topográfico de la sub-cuenca tiene un valor inferior a $40 \mathrm{~m}$, debido al predominio de pendientes menores de $40 \%$ y de longitudes de rampa medias a bajas.

Potencial natural de erosión (PNE). Según la Tabla 8, las áreas con riesgo potencial a la erosión comprende el $82.94 \%$ de la sub-cuenca, de lo cual el $19.92 \%$ tiene un potencial de erosión extremadamente alto, mientras que las áreas menos susceptibles representan sólo el
$17.06 \%$. En general, la sub-cuenca puede presentar serios problemas de erosión, debido a su PNE por encontrarse dentro del rango de moderado a extremadamente altoen su mayor extensión, sobre todo, sin tener en cuenta la posible acción antrópica y la falta de prácticas de conservación para el manejo del suelo.

Uso, manejo y prácticas de conservación de suelos (Factor CP). Según los valores de C y P presentados en la Tabla 3, los valores del factor $\mathrm{CP}$ en agricultura varían de 0.1260 a 0.3600 y en pastizales de 0.004 a 0.01 .

Mapa de uso y ocupación de suelo (MUO). El mapa de uso y ocupación de tierras (MUO) de la sub-cuenca, Fig.5, obtenido por clasificación digital de imágenes CBERS y uso del software SPRING 5.1.6, los resultados se muestran en la Tabla 7 .
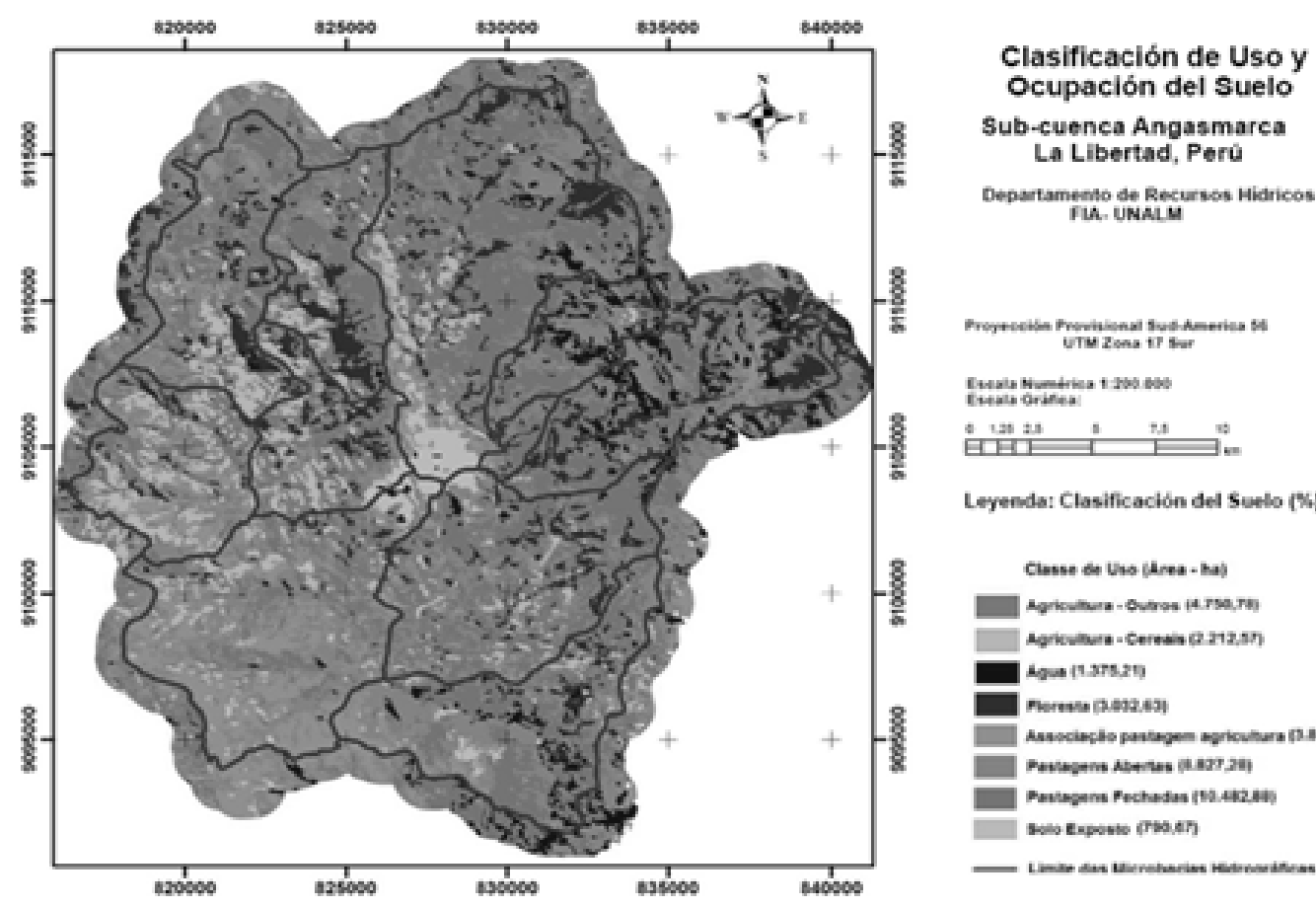

Leyenda: Clasificación del Suelo (\$) Clesse de Uno (lines - has)

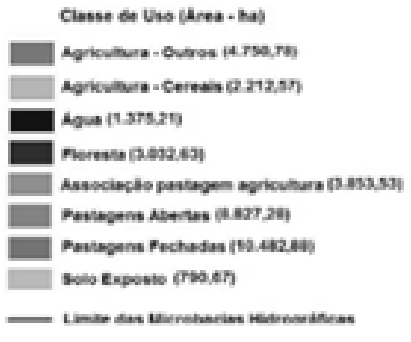

Figura 5. Mapa de uso e ocupación de tierras de la sub-cuenca del ríoAngasmarca, mediante el software SPRING 5.1.6 (INPE). 
DIAGNÓSTICO DEL POTENCIAL DE EROSIÓN HIDRÍCA MEDIANTE TÉCNICAS DE GEOPROCESAMIENTO EN LA SUB-CUENCA DEL RÍO ANGASMARCA,LA LIBERTAD, PERU

Julio-Diciembre 2015

Tabla 6. Longitud de pendiente (L), pendientes (S) y Factor topográfico (LS).

\begin{tabular}{cccccc}
\hline $\begin{array}{c}\text { Micro } \\
\text { cuenca }\end{array}$ & Área & $\begin{array}{c}\text { Longitud } \\
\text { red de drenaje }\end{array}$ & $\begin{array}{c}\text { Longitud } \\
\text { de pendiente }\end{array}$ & $\begin{array}{c}\text { Pendiente } \\
\text { media }\end{array}$ & Factor topográfico \\
\hline & $\mathrm{A}\left(\mathrm{km}^{2}\right)$ & $\mathrm{I}(\mathrm{km})$ & $\mathrm{L}(\mathrm{m})$ & $\mathrm{S}(\%)$ & LS (adim $)$ \\
1 & 52.867 & 30.073 & 439.48 & 31.94 & 27.11 \\
3 & 25.922 & 19.453 & 333.12 & 29.23 & 20.51 \\
4 & 19.793 & 7.596 & 651.37 & 28.05 & 29.80 \\
5 & 31.205 & 18.420 & 423.52 & 28.55 & 23.20 \\
6 & 22.257 & 12.085 & 460.41 & 36.38 & 32.56 \\
7 & 66.140 & 34.652 & 477.16 & 33.54 & 30.25 \\
8 & 15.436 & 12.223 & 315.71 & 49.44 & 36.86 \\
9 & 33.357 & 19.472 & 428.25 & 50.28 & 45.57 \\
10 & 3.126 & 5.062 & 154.40 & 50.69 & 24.19 \\
11 & 46.016 & 23.444 & 490.68 & 35.34 & 32.75 \\
\hline Total & 36.957 & 15.599 & 592.27 & 50.23 & 55.82 \\
\hline Media & 353.075 & 198.085 & - & 38.52 & - \\
\hline
\end{tabular}

Tabla 7. Clasificación de uso y ocupaciónde tierras, sub-cuenca Angasmarca.

\begin{tabular}{crrrrrrrrr}
\hline \multirow{2}{*}{ MCH } & Agua & $\begin{array}{c}\text { Suelo } \\
\text { Expuesto }\end{array}$ & Bosque & $\begin{array}{c}\text { Agricultura } \\
\text { de otros C. }\end{array}$ & $\begin{array}{c}\text { PastizaL } \\
\text { Abierto }\end{array}$ & $\begin{array}{c}\text { Pastizal } \\
\text { cerrado }\end{array}$ & $\begin{array}{c}\text { Agricult. } \\
\text { Cereales }\end{array}$ & $\begin{array}{c}\text { Asoc. Agric- } \\
\text { Past }\end{array}$ & $\begin{array}{c}\text { AREA } \\
\text { TOTAL }\end{array}$ \\
\cline { 2 - 9 } & \multicolumn{1}{c}{ (ha) } & \multicolumn{1}{c}{ (ha) } & \multicolumn{1}{c}{ (ha) } & \multicolumn{1}{c}{ (ha) } & \multicolumn{1}{c}{ (ha) } & \multicolumn{1}{c}{ (ha) } & \multicolumn{1}{c}{ (ha) } & (ha) & (ha) \\
\hline 1 & 41.08 & 205.14 & 93.17 & 586.15 & $1,707.20$ & 631.74 & 324.54 & $1,697.72$ & $5,286.74$ \\
2 & 1.79 & 0.00 & 138.85 & 584.81 & 540.14 & 253.90 & 567.22 & 505.46 & $2,592.18$ \\
3 & 17.53 & 7.00 & 193.60 & 377.18 & 494.09 & 503.95 & 207.29 & 178.61 & $1,979.26$ \\
4 & 27.64 & 11.03 & 305.24 & 594.67 & 779.00 & 794.54 & 326,81 & 281.61 & $3,120.53$ \\
5 & 19.34 & 0.00 & 365.67 & 514.95 & 357.21 & 673.16 & 186.29 & 109.07 & $2,225.71$ \\
6 & 188.04 & 359.20 & 706.51 & 843.76 & $1,237.74$ & $2,666.93$ & 329.76 & 282.02 & $6,613.96$ \\
7 & 276.63 & 11.36 & 114.39 & 12.19 & 490.51 & 629.44 & 0.00 & 9.06 & $1,543.58$ \\
8 & 460.52 & 0.00 & 557.68 & 66.33 & 865.77 & $1,259.72$ & 8.61 & 117.04 & $3,335.67$ \\
9 & 4.76 & 4.16 & 22.06 & 3.68 & 93.28 & 170.35 & 0.00 & 14.33 & 312.63 \\
10 & 104.71 & 108.07 & 368.71 & 899.87 & $1,056.79$ & $1,564.62$ & 192.36 & 306.47 & $4,601.60$ \\
11 & 233.16 & 84.69 & 166.75 & 267.17 & $1,205.55$ & $1,334.54$ & 69.68 & 334.13 & $3,695.67$ \\
\hline Total & $1,375.21$ & 790.67 & $3,032.63$ & $4,750.78$ & $8,827.28$ & $10,482.88$ & $2,212.57$ & $3,835.53$ & $35,307.53$ \\
\hline
\end{tabular}

Tabla 8. Potencial natural de erosión de las micro cuencas del río Angasmarca.

\begin{tabular}{|c|c|c|c|c|c|}
\hline \multirow[b]{2}{*}{$\begin{array}{l}\text { Micro } \\
\text { cuenca }\end{array}$} & \multirow{2}{*}{$\begin{array}{c}\mathrm{R} \\
\text { MJ.mm } \\
\text { ha.h }\end{array}$} & \multicolumn{2}{|c|}{ KLS } & \multirow{2}{*}{$\begin{array}{l}\text { PNE } \\
\frac{t .}{\text { ha.año }} \\
\end{array}$} & \multirow{2}{*}{$\begin{array}{c}\text { Pérdida } \\
\text { de suelos } \\
\left(\text { t.ha }{ }^{-1} \cdot \text { ano }^{-1}\right)\end{array}$} \\
\hline & & $\frac{\text { t.ha.h }}{\text { ha.MJ.mm }}$ & Adim. & & \\
\hline 1 & $4,762.00$ & 0.03412 & 27.11 & $4,404.82$ & 81.37 \\
\hline 2 & $4,762.00$ & 0.03412 & 20.51 & $3,332.45$ & 65.48 \\
\hline 3 & $4,762.00$ & 0.03412 & 29.80 & $4,841.89$ & 93.29 \\
\hline 4 & $4,762.00$ & 0.03412 & 23.20 & $3,769.52$ & 72.63 \\
\hline 5 & $4,762.00$ & 0.03412 & 32.56 & $5,290.33$ & 94.39 \\
\hline 6 & $4,762.00$ & 0.03412 & 30.25 & $4,915.00$ & 93.50 \\
\hline 7 & $4,762.00$ & 0.03412 & 36.86 & $5,988.99$ & 106.60 \\
\hline 8 & $4,762.00$ & 0.03412 & 45.57 & $7,404.19$ & 133.59 \\
\hline 9 & $4,762.00$ & 0.03412 & 24.19 & $3,930.38$ & 69.96 \\
\hline 10 & $4,762.00$ & 0.03412 & 32.75 & $5,321.20$ & 98.67 \\
\hline 11 & $4,762.00$ & 0.03412 & 55.82 & $9,069.60$ & 166.52 \\
\hline Total & $4,762.00$ & 0.03412 & 35.80 & $5,816.76$ & $1,075.99$ \\
\hline
\end{tabular}


Pérdida de suelo por unidad de área (A) en t.ha $\mathbf{a n o}^{-1}$. Según los resultados en la Tabla 8 y la Fig.6, la distribución espacial de la susceptibilidad a la erosión está dada por el relieve, el uso y ocupación del suelo de la sub-cuenca. Las micro cuencas con estimaciones superiores a 97.82 t.ha ${ }^{-1} a_{n} o^{-1}$, se encuentran en zonas de mayor pendiente en las divisorias de las micro cuencas, laderas y crestas de los cerros, en un área de $88.88 \mathrm{~km}^{2}$. Sin embargo, en las micro cuencas donde ocurren menores pérdidas de suelos, de 77.47 t.ha ${ }^{-1} a_{n ̃ o}^{-1}\left(76.92 \mathrm{~km}^{2}\right)$, se encuentran en terrenos con pendientes planas a moderadas. Las pérdidas promedio de suelo en la sub-cuenca de 97.82 t.ha ${ }^{-1} \mathrm{año}^{-1}$, se caracteriza como pérdida de suelo "media fuerte", según

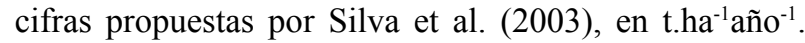
Los resultados muestran que los suelos con menores tolerancias de pérdidas por erosión son los Xerosoles lúvico y Páramo Andosoles con 10.87t.ha ${ }^{-1} \mathrm{año}^{-1}$ y 13.60 t.ha ${ }^{-1} \mathrm{año}^{-1}$, respectivamente, pero mostró pérdidas reales de suelo de 50.68 a 58.35 t.ha $^{-1}$.año ${ }^{-1}$. En el caso de suelos Kastanazem lúvicos y cálcicos la tolerancia media en el horizonte A es de 23.21t.ha ${ }^{-1}$ año $^{-1}$ para pérdidas efectivas promedio de 50.02 t.ha- año $^{-1}$.

Tabla 9. Potencial natural de erosión para la sub-cuencaAngasmarca, Segúnadaptaciónde Bertoni \& Lombardi N. (2010); Scopel (1988) yNascimento (1998).

\begin{tabular}{|c|c|c|c|c|}
\hline $\begin{array}{c}\text { Potencial de } \\
\text { Pérdidasde suelo }\end{array}$ & $\begin{array}{l}\text { PNE=R.K.LS } \\
\left(\mathrm{t} \cdot \mathrm{ha}^{-1} \cdot \mathrm{ano}^{-1}\right)\end{array}$ & $\begin{array}{l}\text { Micro } \\
\text { cuenca }\end{array}$ & $\begin{array}{l}\text { Área } \\
\left(\mathrm{km}^{2}\right)\end{array}$ & $\begin{array}{c}\text { Área } \\
\text { Total }(\%)\end{array}$ \\
\hline Moderado & $3,000.0-4,000$ & $2,4,9$ & 60.253 & 17.06 \\
\hline Medio & $4,000.1-5,000$ & $1,3,6$ & 138.800 & 39.31 \\
\hline Alto & $5,000.1-6,000$ & 7,10 & 83.709 & 23.71 \\
\hline Muy alto & $6,000.1-7,000$ & -------- & --------- & -------- \\
\hline Extremamente alto & $7,000.1-8,000$ & 8 & 33.357 & 9.45 \\
\hline Extremamente muy alto & $>8,000.1$ & 11 & 36.957 & 10.47 \\
\hline Total & ----------- & --------- & 353.075 & 100.00 \\
\hline
\end{tabular}

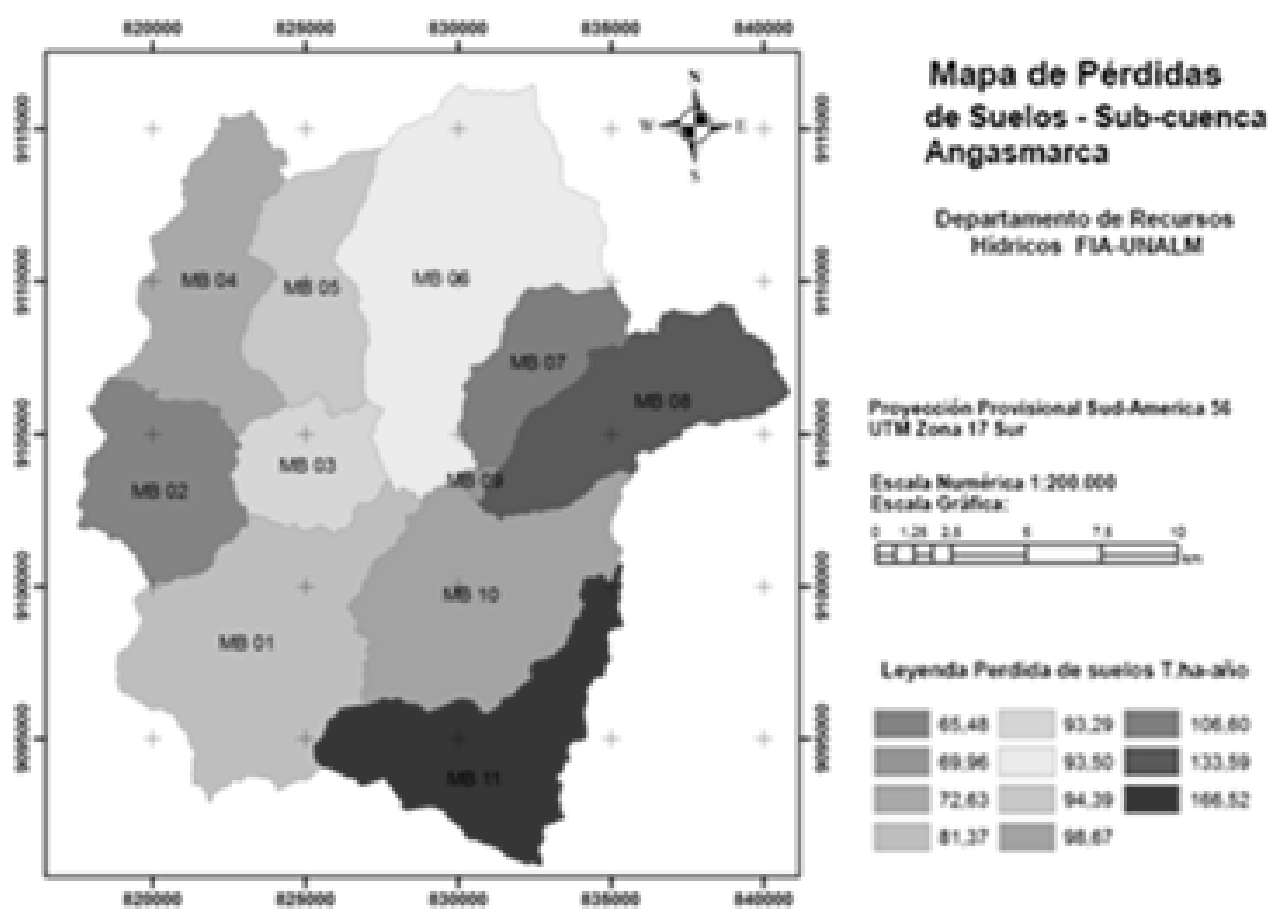

Figura 6. Mapa de riesgos de erosión o de pérdidas de suelos por erosión hídrica Acciones propuestas para mitigar zonas con riesgo de erosión. 
Algunas recomendaciones para zonas con riesgos significativos de erosión:

1. Plantío en nivel: dirigido a la prevención o reducción de pérdida de suelos, agua y fertilizantes, por erosión laminar, optimiza las operaciones con maquinaria agrícola.

2. Adopción del sistema de labranza que no promuevan pulverización excesiva del suelo (labranza, mínima o sembrío directo) y dejar todo el material residual en la superficie e incorporar al suelo.

3. El mantenimiento o mejora de las condiciones físicas del suelo: adopción de rotación de cultivos, aumento de la productividad combinado con la conservación del suelo.

4. Pisos de vegetación permanentes: promueve la cubierta vegetal que evite la erosión (proporcionan forraje, semillas, alimentos y la protección de la vida silvestre).

5. Distribución de animales por unidad de área, destacando su capacidad de soporte al pastoreo, pastoreo rotacional.

6. Enriquecer el bosque: la forestación debe ser heterogénea y contener al menos un $50 \%$ de las especies nativas.

7. Mantenimiento anual de las terrazas existentes. 8.Educación ambiental.

9. Facilitar y supervisar la regeneración natural de la vegetación.

10. Prohibición de cualquier tipo de concesión minera.

\section{Conclusiones}

Es posible llevar a cabo la evaluación ambiental en la sub-cuenca del río Angasmarca utilizando técnicas de geoprocesamientoa partir de la espacialización de la información y la realización de los análisis ambientales.

Caracterización morfométrica de la sub-cuenca relacionada con el sistema de drenaje y relieve con pendientes medias altas indican que el área de estudio está potencialmente sujeto a los fenómenos de erosión, especialmente en condiciones de suelo expuesto o de escasa cobertura vegetal permanente, también en áreas donde se practican formas de agricultura que protegen poco y exponen el suelo más a la erosión, así como por el manejo no adecuado de pastizales.

Las áreas con mayor susceptibilidad a la pérdida potencial de suelos por erosión laminar de la aplicación del modelo matemático de USLE, son las micro cuencas hidrográficas tienen altos grados de pendientes, sin embargo, sin sobre estimar el factor topográfico, el análisis de la pérdida de suelos por erosión laminar considera todos los factores de la USLE.
Los modelos matemáticos realizados en Sistemas de Información Geográfica (SIG), posibilitan la integración de datos, la estimación del potencial erosión laminar y permite espacializar áreas de riesgo potencial delsuelo a la erosión y la degradación del medio ambiente.

Las medidas de mitigación propuestas para las áreas que presentan riesgos o limitaciones de uso del suelo significativas, se presentan en forma de recomendaciones técnicas para que los impactos ambientales del uso indiscriminado de estas tierras para que tengan su magnitud reducida.

\section{Literatura citada}

Bertoni, J. y Lombardi, N. F. 2010. Conservação do solo - São Paulo: Icone, $7^{\mathrm{a}}$ edição. $355 \mathrm{p}$

Cabral, J. B. P. et al. 2005. "Estudo da erosividade e espacialização dos dados com técnicas de Geoprocesamiento na carta topográfica de Morrinhos Goiás/Brasil para o período de 1971 a 2000”, GeoFocus (Artículos), No 5, p. 1-18. ISSN: 1578-5157.

Christofoletti, A. 1999. Modelagem de sistemas ambientais. São Paulo: Edgard Blucher, 236 p.

Djokic, D.; Ye, Z. and Miller. A. 1997. Efficient Watershed Delineation Using ArcView and Spatial Analyst, Proc. 17th Annual Esri User Conference, San Diego, CA.

ESRI (Environmental Systems Research Institute). 1997. Watershed Delineator Application -User's Manual. Redlands, CA.

Farinasso, M. 2005. Avaliação qualitativa do potencial erosivo em grandes áreas por meio da EUPS-Equação Universal de Perdas de Solos utilizando novas metodologias Dissertação. Mestrado em Geografia. Instituto de Ciências Humanas, Universidade de Brasília, Brasília, $105 \mathrm{f}$.

Guerra, A. J. T. y Cunha, S. B. 1996. Degradação ambiental. Geomorfologia e meio ambiente. Rio de Janeiro: Bertrand Brasil, p. 93 199, 1994 y p. 337-379.

INPE (Instituto Nacional de Pesquisas Espaciais). Julio 2011. Ministério de Ciência Tecnologia e Inovação, Unidade de produtos e serviços, engenharia de satélites CBERES, http://www.inpe.org.br.

Nascimento, M. A. 1998. Bacia do rio João Leite: influência das condições ambientais naturais e antrópicas na perda de terra por erosão laminar. Tese de Doutorado em Geografia. Instituto de Geociências e Ciências Exatas, Universidade Estadual Paulista Hulio Mesquita Filho, Rio Claro. $176 \mathrm{f}$.

Olivera, F. P. 2004.Determinación de la Tolerancia de pérdidas por erosión para las principales ordenes de suelos del Estado de Paraíba. Monografía (trabajo de pregrado en ingeniería Agronómica)- Centro de Ciencias Agrarias, Universidad Federal de Paraíba, Arena. 92f. 
PERU, Ministerio de Agricultura-ANA, 2011.http:// www.ana.gob.pe/marco-normativo. aspx.

PERU, ONERN (Oficina Nacional de Evaluación de Recursos Naturales). 1972. Inventario evaluación y uso racional de recursos naturales de las cuencas hidrográficas de Santa, Nepeña y Lacramarca, Lima, Perú.

Pruski, F. F.; Amorim, R. S. S.; Silva, D. Dom; Griebeler, N. P. y Silva, J. M. A. 2006. Conservação de solo e água: praticas mecânicas para o controle da erosão hídrica. Viçosa: Universidade Federal de Viçosa, 240 p.

Pruski, F. F. 2009. Conservação de solo e água: praticas mecânicas para o controle da erosão hídrica. Viçosa: Universidade Federal de Viçosa, 2a edição, 279 p.

Ribeiro, F. L. 2002. Mapa de vulnerabilidade à erosão da região do Alto Rio Pardo - Pardinho (SP). Tese (Doutorado em Agronomia) - Faculdade de Ciências Agronômicas, Universidade Estadual Paulista Júlio de Mesquita Filho, Botucatu, $119 \mathrm{f}$.

Righetto, A. M. 1998.Hidrologia e Recursos Hídricos. São Paulo: EESC/USP, p. 731 - 813.

Ruhoff, A. L.2004. Gerenciamento de recursos hídricos - Modelagem ambiental com a simulação de cenários preservacionistas. Dissertação (Mestrado em Geomática). Santa Maria: UFSM.

Tomazoni, J. C. y Guimarães, E. A. 2008. Sistematização dos fatores da USLE em SIG para quantificação da erosão laminar na bacia do rio Jirau. Revista Brasileira de Cartografia, Rio de Janeiro,v. 57,n. 3, Disponível em: http://www.rbc.ufrj.br/_20057_3_07. Htm > Acesso em: 05 maios. 